\title{
RF MEMS Switches for Smart Antennas
}

\author{
Jitendra Pal $\cdot$ Yong Zhu $\cdot$ Junwei Lu $\cdot$ Dzung Viet Dao
}

\begin{abstract}
In this paper novel V-shaped and Z-shaped thermally actuated Radio Frequency (RF) MicroElectroMechanical Systems (MEMS) switches are designed and fabricated for the application of smart antennas. The switches are driven by a metal electrothermal actuator, which is able to generate large displacement and high contact force at lower temperatures. The MEMS switches utilizing the parallel beam actuator achieved $8 \mu \mathrm{m}$ displacement. RF performances are improved by suspending the switching structures $25 \mu \mathrm{m}$ above the substrate, thereby reducing the loss in the substrate. ON state insertion loss of $-0.42 \mathrm{~dB}$ at $10 \mathrm{GHz}$, OFF state isolation of $-40 \mathrm{~dB}$ at $10 \mathrm{GHz}$ and return loss better than of $-20 \mathrm{~dB}$ at $10 \mathrm{GHz}$ for bidirectional Z-shaped thermally actuated RF MEMS switch are achieved on low resistivity silicon substrate.
\end{abstract}

\section{Introduction}

With the tremendous advancement in communication technology, new technologies are being investigated to improve performances and usage of the available spectrum in the most efficient way. Smart antennas with controllable directionality are promising candidate as they allow higher reuse of channels and increased system performances. One of the most challenging aspects of smart antenna design is inclusion of the necessary switching circuitry that is done with bulky passive components. However, some off-chip bulky passive element such as varactor or PIN diodes, inductors, quartz crystals, and SAW filters, have become limiting for the chip scaling down (H. A. C. Tilmans et al. 2003). Hence, RF MEMS featuring small size, low weight and high performance can be considered as a future enabling technology to replace off-chip passive elements (D. Girbau et al. 2006).

In previous work, PIN Diodes were used for the RF switching for a Patch Antenna with Thirteen Hexagonal Element (PATHE) array operating at frequency range of
2.4-5.8 GHz and Dielectric-Embedded Electronically Switched Multiple-Beam (DE-ESMB) as illustrated in Fig. 1 and Fig. 2 (J. Lu et al. 2002). However, PIN diodes have lower $\mathrm{Q}$ factor at high frequencies and therefore high insertion loss. Compared with the PIN Diodes, MEMS switches offer higher $\mathrm{Q}$ factor at high frequencies, low insertion loss, higher isolation and smaller packaging size. Also, the idea of System on Chip (SOC) becomes more realistic in the communication system due to compatibility of MEMS switch with the IC process. Hence, the integration of MEMS switches into RF subsystems is expected to provide benefits.

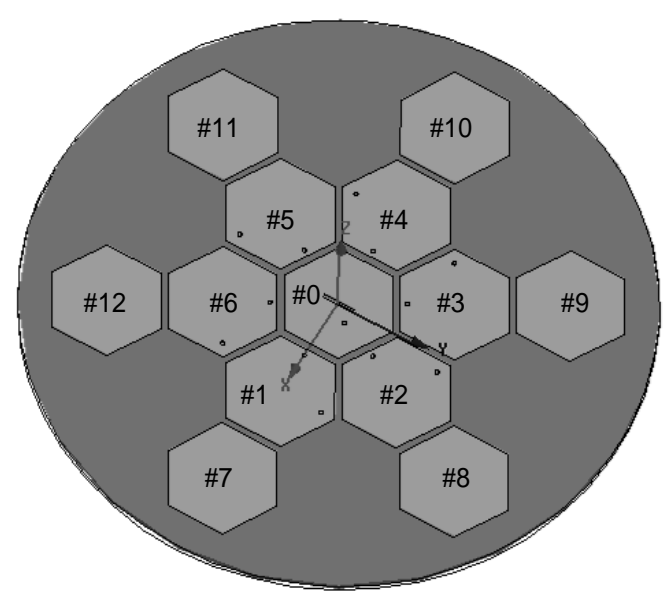

Fig. 1 Layout for the single-feed circularly polarized patch antenna array

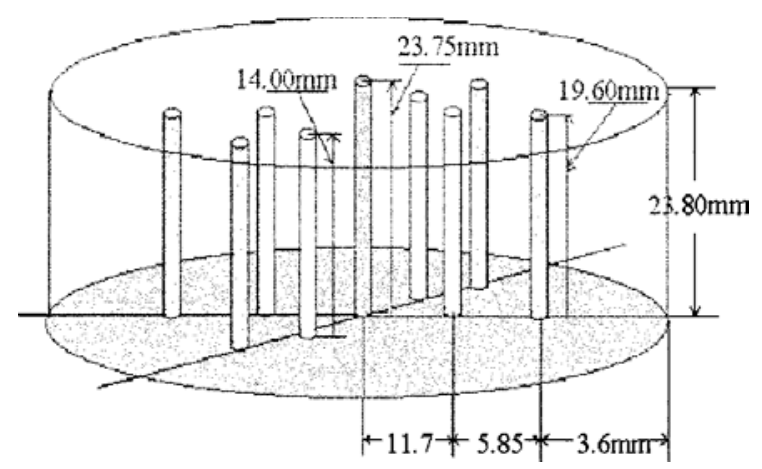

Fig. 2 Configuration of DE-ESMB antenna array 
Various actuation approaches have been demonstrated utilizing various operation principles such as the electrothermal actuation, the electrostatic actuation, the piezoelectric actuation, and the electromagnetic actuation. Among all of these principles, the electrostatic and the electrothermal actuators are most attractive. The electrostatic actuators offer low power dissipation and high driving frequency, but suffer from less functional robustness, high actuation voltage and small range of controllable displacement. In contrast, the electrothermal actuators have capability in generating relatively large actuation displacement and force, but a relatively high power consumption as compared with the electrostatic actuators (W. Shi et al. 2007). Therefore, the actuation method should be selected according to the application requirements of the switches.

In this paper, we introduce V- shaped and Z-shaped thermal actuators that are used to replace PIN Diodes for switching purposes. The proposed RF MEMS switches are implemented by using MetalMumps process (A. Cowen et al. 2007), a commercially available Multi Project Wafer (MPW) service. Through MetalMumps process the substrate loss $(25-\mu \mathrm{m}$ deep trench is carved under the device, resulting in a suspended switch) can be reduced substantially. Also, it can provide a good solution for the integration of MEMS with IC. Hence, it is able to bring the concept of System on Chip (SOC) into reality.

\section{Working Principle of Antennas}

\subsection{Thirteen Hexagonal Element Antenna Array}

Earlier two separate driven sources and a power splitter were used to generate two feeds for the driven element patch to create circular polarisation (W. H. Chen et. al. 2007). Building upon this design as shown in Fig. 1, we developed a patch antenna with thirteen hexagonal element (PATHE) arrays. To increase antenna gain in each switched direction six additional parasitic elements with the central driven element surrounded by six hexagonal parasitic elements (\#1 - \#6) in the same configuration as PASHE having equal size and shape were used. An additional six elements (\#7 - \#12) were placed in an outer circle which acts as director elements.

Fig. 3 illustrates switched beam-forming structure for the single-feed circularly polarized patch antenna array.
During operation, all outer ring elements (\#7 - \#12) and one inner ring element (\#1) function as director elements and the remaining elements in inner ring are shortcircuited to ground.

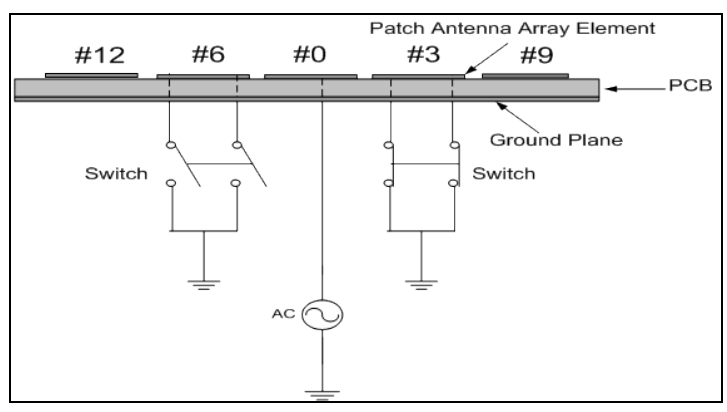

Fig. 3 A typical dual switch beam-forming circuit for the single feed circularly polarized patch antenna array

\subsection{DE-ESMB antenna array}

DE-ESMB antenna array can produce two beams simultaneously that are separated by $180^{\circ}$ with four possible beam directions at $90^{\circ}$ spacing through the azimuthal plane. It gives the characteristics of Yagi-Uda antenna array by sorting to the ground plane to the center elements of the antenna array. The centre element is slightly longer than a resonant quarter wavelength element and surrounded by elements placed along two radially concentric circles. These elements can be switched to a number of possible different states. The inner circle of elements may be either active elements or passive elements. If these are passive elements then they can be shorted to ground or isolated from the ground plane. The outer circle of elements are always parasitic, but can be switched from open circuit to short circuit. Each circle has the same number of elements. The switching is achieved using a novel bidirectional Zshaped thermally actuated RF MEMS switch as shown in Fig. 4.

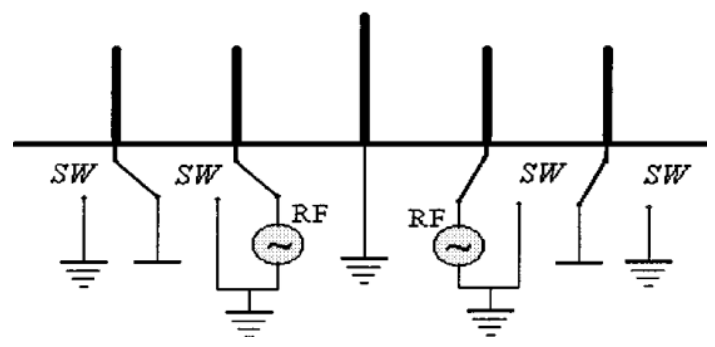

Fig. 4 Switching circuits for beam control in DE-ESMB antenna array 


\section{Design of RF MEMS Switches}

3.1 Design of V-shaped thermally actuated RF MEMS switch for a patch antenna with thirteen hexagonal element (PATHE) arrays

\subsubsection{Operational Principle}

Fig. 5 shows the operational principle of thermally actuated MEMS switch. A V-shaped actuator, which is made of pre-curved nickel beams, is employed to provide lateral motion in the wafer plane. This switch adopted a piece of silicon nitride as an electrical isolation layer between the driving structure and contact structure. When a current flows through the beams, the electrothermal actuator generates movement due to expansion of its material and allows contact heads to move forward and short signal lines to ground as illustrated in Fig. 5(b). On removing the current, the switch returns back to its original position due to internal restoring force of the beam, as illustrated in Fig. 5(a).

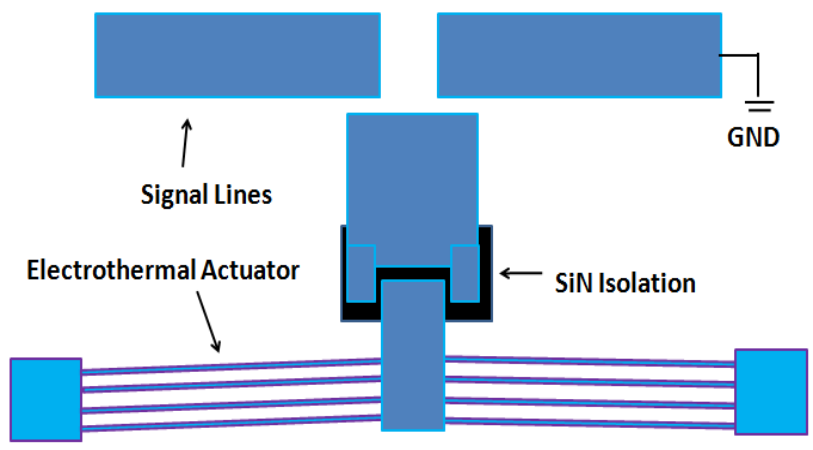

(a) OFF

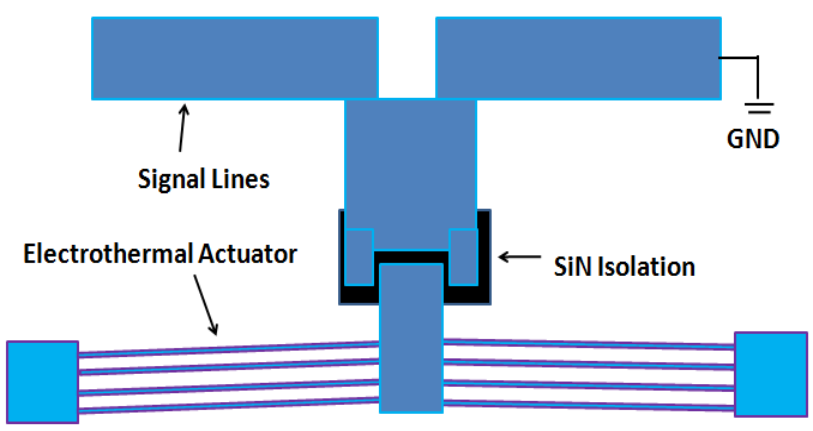

(b) $\mathrm{ON}$

Fig. 5 Working principle of $V$-shaped thermally actuated MEMS switch

\subsubsection{Actuator Design}

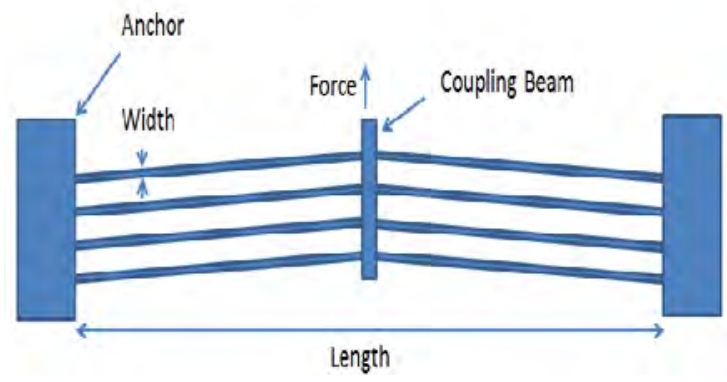

Fig. 6 Schematic diagram of the V-shaped parallel beams thermal driven structure

Thermal actuators are very good candidates for RF MEMS switches as they offer high contact force and low contact resistance. In this work, V-shaped electrothermal actuator beams are chosen for their rectilinear displacement caused by joule heating. The design and flexibilities are as illustrated in Fig. 6. The beam is designed with careful considerations such that temperature is below $450^{\circ} \mathrm{C}$ to prevent plastic deformation and surface oxidation [J. K. Luo et al. 2009]. Higher force is needed to allow better contacts and lower metallic losses, which translate in to lower switch insertion loss. Therefore, following equations were utilized, while designing the structure (M. J. Sinclair 2000).

$$
\begin{gathered}
d=\left[l^{2}+2(l) l^{\prime}-l \cos (\theta)\right]^{1 / 2}-l \sin (\theta) \\
F=N \frac{E w^{3} t}{4 l^{3}} d
\end{gathered}
$$

Where

$\mathrm{d}:$ actuator displacement

$1:$ length of the beam

1 ' : elongation of the beam due to thermal expansion

$\theta:$ pre-bended angle of the beam

$\mathrm{F}$ : applied force

$\mathrm{N}$ : Number of actuators

E : Young Modulus

$\mathrm{t}$ : beam thickness

$\mathrm{w}$ : beam width

According to the above equations, it can be concluded that device performances can be improved by changing 
the structure of the beam. At a given temperature, the beam displacement can be increased by increasing the beam length or reducing the pre-bended angle. The actuators exhibit an output force which is directly affected by the thickness and width of the beam (M. J. Sinclair 2000).

Nickel is chosen for the actuator building material as figure of merit of nickel $Q=\alpha / k E$ where $\alpha$ is the thermal expansion coefficient, $k$ is the thermal conductivity, and $E$ is the Young modulus, is better than polysilicon. Also, thermal expansion coefficient of nickel as illustrate in Table 1 is high compared with polysilicon, therefore nickel beams are able to achieve same displacement at a lower temperature in comparison with polysilicon beams [D. Girbau et. al. 2007].

TABLE 1

Properties of Polysilicon and Nickel

\begin{tabular}{lll}
\hline Parameters & Poly-Si & Nickel \\
\hline$\alpha(\mathrm{ppm} / \mathrm{K})$ & 2.5 & 12 \\
$k(\mathrm{~W} / \mathrm{mK})$ & 65 & 91 \\
$E(\mathrm{Gpa})$ & 165 & 210 \\
$Q$ & $2.33 \times 10^{-13}$ & $6.28 \times 10^{-13}$ \\
\hline
\end{tabular}

The area of proposed microswitch is approximately $1000 \mu \mathrm{m} \times 470 \mu \mathrm{m}$. Dimension of a single $\mathrm{V}$-shaped beam is $1000 \mu \mathrm{m}$ in length, $10 \mu \mathrm{m}$ in width, $20 \mu \mathrm{m}$ in thickness, $10 \mu \mathrm{m}$ offset at the centre, and $1.05^{\circ}$ pre-bended angle respectively. The RF signal lines are separated from the contact head by $8 \mu \mathrm{m}$ and contact area is designed to be $50 \mu \mathrm{m} \times 20 \mu \mathrm{m}$.

\subsection{Design of bi-directional Z-shaped thermally actuated RF MEMS switch for Dielectric-Embedded Electronically} Switched Multiple-Beam (DE-ESMB)

\subsubsection{Z-shaped Bi-directional Thermal Actuator}

The V-shaped thermal actuators have been employed in many applications as they can easily generate large force (on the order of $\mathrm{mN}$ ). However, they have some limitations such as slanted beams, which pose challenges for fabricating small features with smooth sidewall surfaces, especially as the beam width gets close to the resolution of photography. The other drawback is that Vshaped electrothermal actuators beams posses an extremely high mechanical stiffness when forced against their direction of operation, rendering them unsuitable for bidirectional motion [Y. Zhu et. al. 2005].

These limitations can be overcome using Z-shaped thermal actuator which is connected back- -to-back to achieve bidirectional actuation without buckling in $V$ shaped beams. Also, it offers a large range of stiffness and output that is complementary to the comb drives and V-shaped actuators [Y. Zhu et. al. 2012].

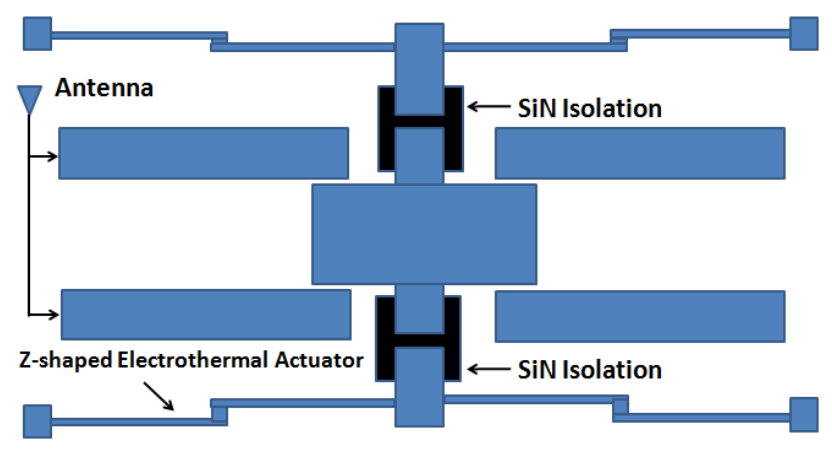

(a) OFF State

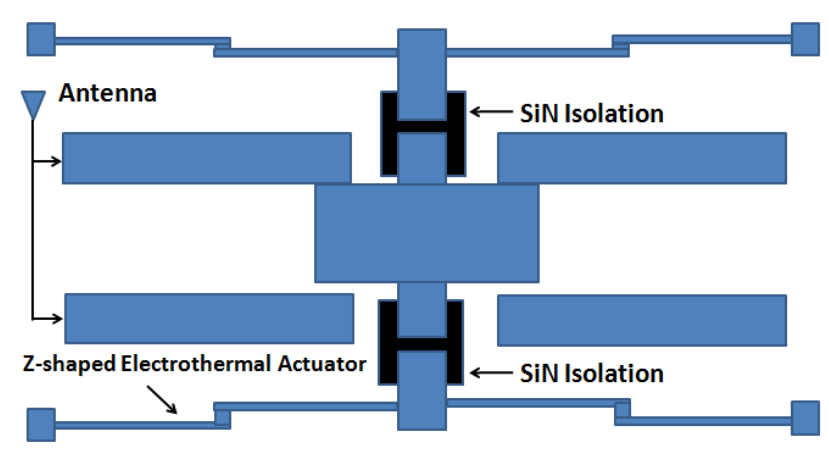

(b) ON state, connect to RF signals

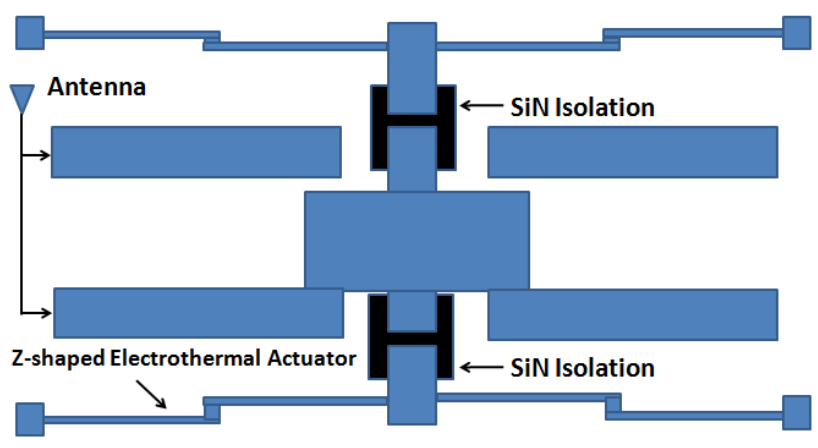

(c) ON state, connect to ground

Fig. 6 Working principle of bi-directional Z-shaped thermal actuator 
Working principle of Z-shaped and V-shaped thermal actuator is almost similar. When a current is passed through the device, heat is generated along the beam due to joule heating. The temperature rise leads to thermal expansion of all beams, especially the long beam. The long beams cannot expand straight due to symmetry of the structure, rather they bend to accommodate length increase. Hence, the shuttle is pushed forward and connects to RF signal lines to turn on the switch as illustrate in Fig. 6 (b). Similarly, the central shuttle will move downward when applying current through other actuators and connect antenna signal to ground as illustrate in Fig. 6(c). On removing the current, the switch returns back to its original position due to internal restoring force of the beam as illustrated in Fig. 6 (a).

\subsection{Design of Coplanar Waveguide (CPW)}

Fig. 7 illustrates the schematic diagram of Coplanar Waveguide (CPW) for a Z-shaped bi-directional thermally actuated RF MEMS switch. The switch consists of a finite ground coplanar waveguide (FGCPW) transmission line and an electrothermal actuator suspended $25 \mu \mathrm{m}$ over the low-resistivity substrate (the separation is defined by the trench). In conventional $\mathrm{CPW}$, much of the electric field distributes in the free space lading higher radiation loss and conduction loss is higher due to crowding of surface current on the edge of the centre conductor. However, in proposed design, a trench of $25-\mu \mathrm{m}$ deep is carved under the device, resulting in a suspended switch. Therefore, propagation losses in the substrate can be reduced as most of the electric field is confined to the air between the lines.

In the proposed structure, the transmission line is designed from three parallel plate waveguides running parallel to one another, which are realized by forming each waveguide from a $20 \mu \mathrm{m}$ thick nickel plate that has been coated with a thin layer of gold. Therefore the RF signal can propagate not only along the metal on the top surface, but also along the metal on the sidewall of the transmission line. With the help of the three dimensional finite element method (3D FEM) simulation software Ansoft's High-Frequency Structure Simulator (HFSS), a $50 \mathrm{ohm}$ transmission line can be obtained by adjusting the width of the FGCPW signal line (S) and the gap between the signal line and the ground line $(\mathrm{W})$. In this switch, the parameters $\mathrm{S}$ and $\mathrm{W}$ were designed to be $100 \mu \mathrm{m}$ and 20 $\mu \mathrm{m}$ respectively to accommodate the $150 \mu \mathrm{m}$ pitch ground-signal-ground coplanar probes.

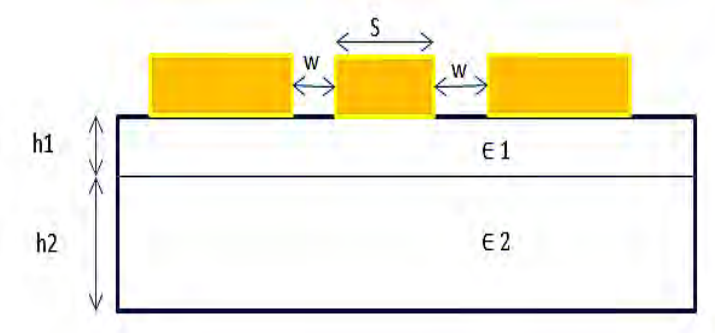

Fig. 7 Schematic diagram of Finite Ground Coplanar Waveguide (FGCPW)

\section{Fabrication Process}

Fig. 8 illustrates the cross-sectional view of fabricated Vshaped and Z-shaped thermally actuated RF MEMS switches. The device is optimized on a low resistivity silicon substrate $(1-2 \Omega-\mathrm{cm})$ using MetalMumps technology, a commercially available multi project wafer process and simulated in virtual clean room simulation module (Fabviewer) in Intellisuite software. The process includes a $2 \mu \mathrm{m}$ thick isolation layer on starting silicon wafer. This is followed by the deposition of a $0.5 \mu \mathrm{m}$ thick sacrificial layer (oxide 1) defining the area where the trench under the device will be patterned at the end of the process. Afterwards, two sequential silicon-nitride layers (Nitride 1 and Nitride 2) are deposited and patterned forming structural connection as well as electrical and thermal isolations between actuator and RF signal lines. Then, access pads are defined in a second sacrificial layer. The wafer is patterned with $20 \mu \mathrm{m}$ thick structural nickel layer into the patterned resist stencil. After electroplating, gold layer is electroplated. Finally, $\mathrm{KOH}$ solution is used to form a $25 \mu \mathrm{m}$ deep trench in the silicon substrate.

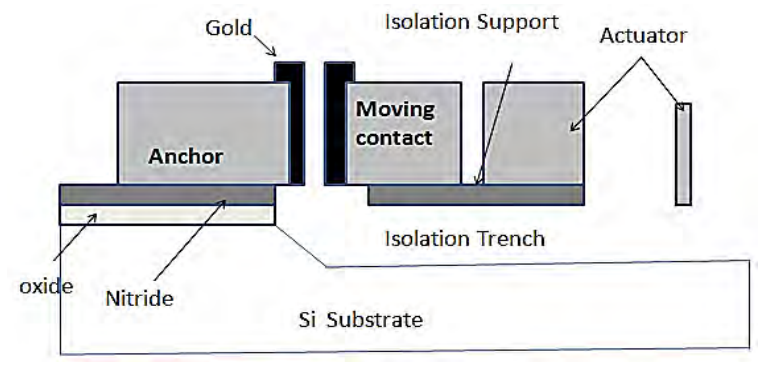

Fig. 8 Cross-sectional view of MEMS switch manufactured with MetalMumps. 


\section{Results}

\subsection{V-shaped Thermally Actuated MEMS Switch}

The SEM photograph of the manufactured V-shaped electrothermal MEMS switch is shown in Fig. 9. This MEMS switch is designed for the application in PATHE antenna array. Fig. 10 illustrates the static displacement and maximum temperature as a function of actuation current for an electroplated $\mathrm{Ni}$ parallel four-beam actuator. It indicates that the displacement and temperature increase with the applied current as expected. For an $8 \mu \mathrm{m}$ maximum displacement an applied current of $1 \mathrm{~A}$ and $102{ }^{\circ} \mathrm{C}$ maximum temperature are required.

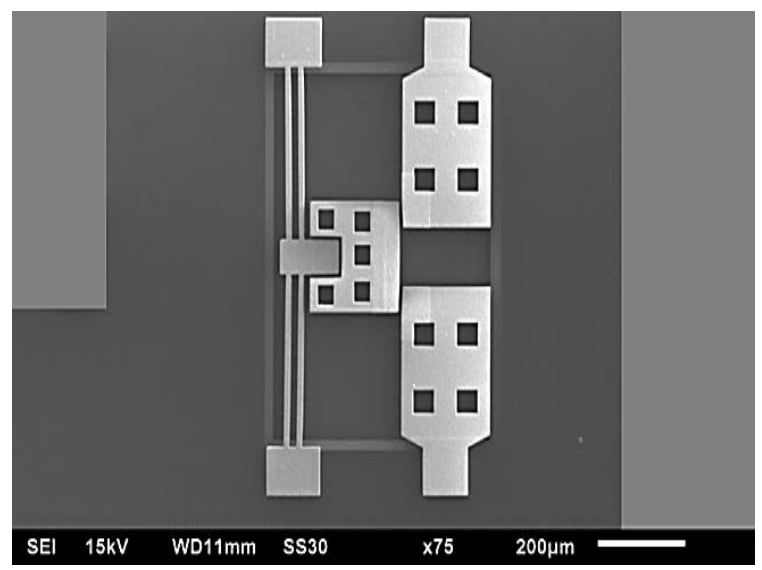

Fig. 9 SEM photograph of a V-shaped thermally actuated RF MEMS switch

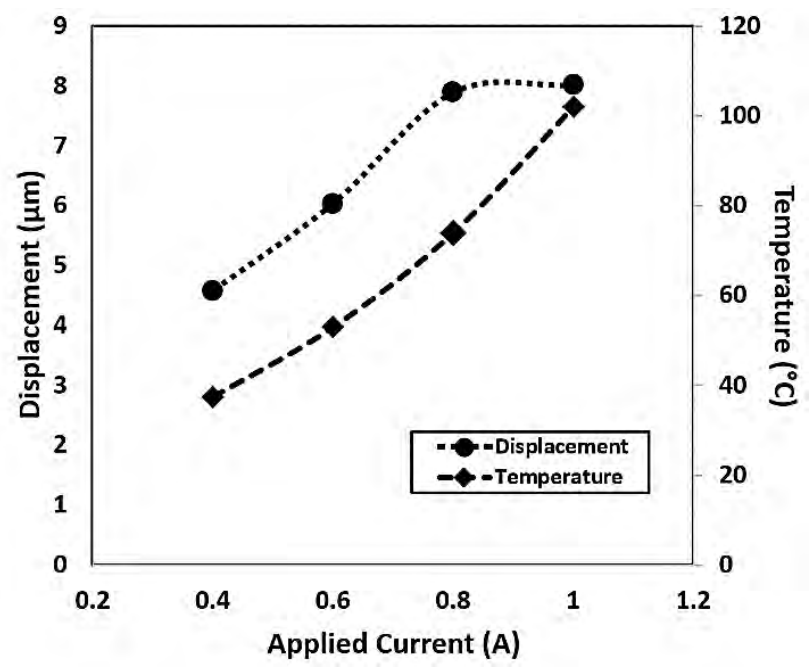

Fig. 10 Displacement and Temperature versus applied current.
Figures 11 - 13 illustrate the simulated displacement, temperature and contact force for V-shaped thermally actuated RF MEMS switch with $1 \mathrm{~mm}$ length, $10 \mu \mathrm{m}$ width, $20 \mu \mathrm{m}$ thickness and $1.05^{\circ}$ pre-bended angle. The structure was simulated using Intellisuite software. The end of the beams were fixed and the boundary was set at the temperature of $25^{\circ} \mathrm{C}$ at the end of the beams. When current is applied at the end of the beams, current flows through the beams and generates heat, thereby generating the movement due to expansion of the beams.

The output force exhibited by the actuators depends on the number of buckle beams, pre-bended angle and actuator layer thickness as described in Eq. (2). Thus, on increasing the number of actuators, the actuators will exhibit more output force. There are no limitations to add numbers of actuators as long as the current and heat can be handled by the device and conductors. However, power consumption will be high on increasing the number of actuators. Fig. 13 illustrates high contact force has been achieved, thus allowing good contacts and low contact resistance.

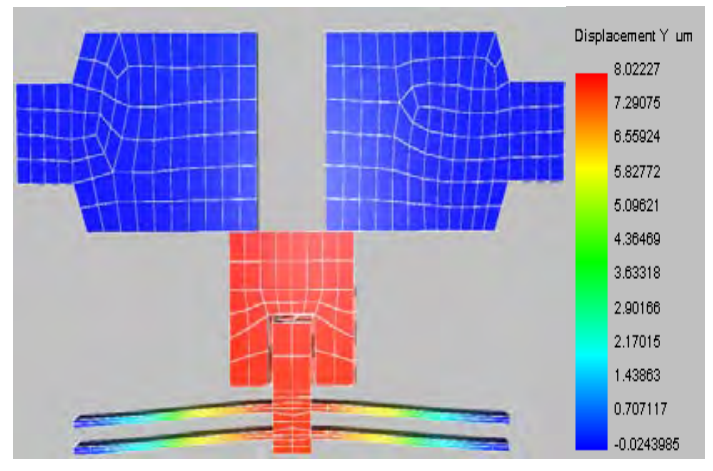

Fig. 11 Displacement with applied current of $1 \mathrm{~A}$.

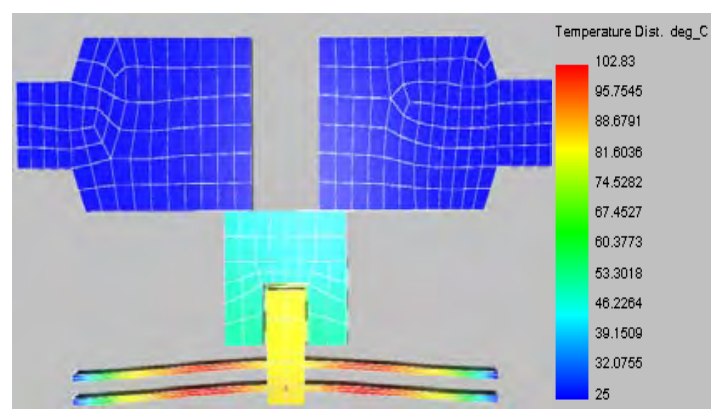

Fig. 12 Temperature distribution with applied current of $1 \mathrm{~A}$. 


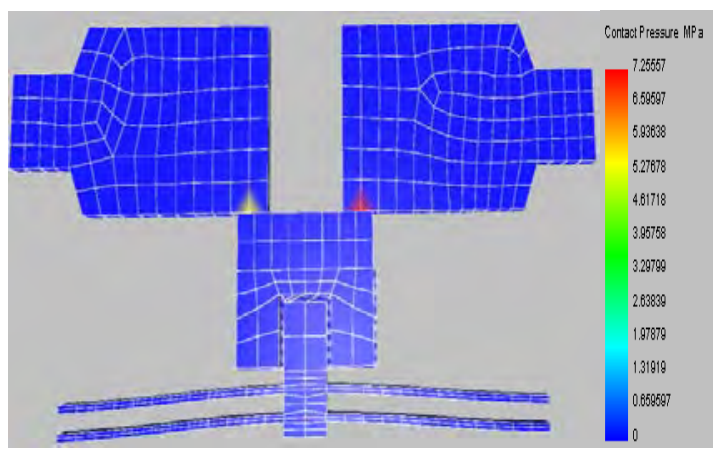

Fig. 13 Contact force with applied current of $1 \mathrm{~A}$.

\subsection{Z-shaped Thermally Actuated MEMS Switch}

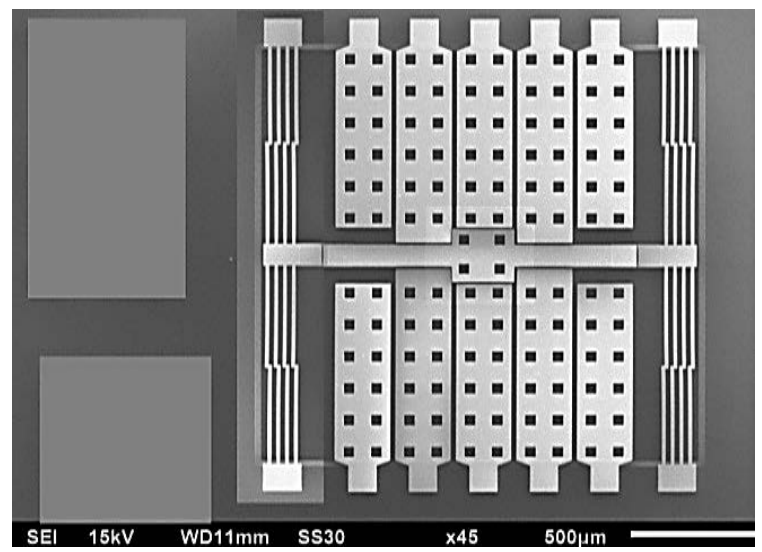

Fig. 14 SEM photograph of a bi-directional Z-shaped thermally actuated RF MEMS switch

The SEM photograph of the manufactured Z-shaped electrothermal MEMS switch is shown in Fig. 14. In this design, Z-shaped electrothermal actuators are used to overcome the limitations of $\mathrm{V}$-shaped thermal actuators, which are able to operate in forward and backward directions, while generating large displacement and high contact force. The designed switch includes RF pads, switch contacts and Z-shaped actuators. The switch is based on a coplanar waveguide transmission line, which is designed with $50 \Omega$ characteristic impedance. The switch is basically used for switching the beam of DEESMB antenna array between ON and OFF states. The switch is based on the principle that when electrical current flows through a beam, the joule heating causes it to expand and allows mechanical motion in specific direction.
Fig. 15 illustrates the static displacement and maximum temperature as a function of actuation current for a fourbeam bi-directional Z-shaped actuator. It can be observed that the displacement and the temperature increase with the increase of the applied current through the beams. The maximum displacement of $8.5 \mu \mathrm{m}$ and maximum temperature of about $68{ }^{\circ} \mathrm{C}$ is obtained on applying actuation current of $1.2 \mathrm{~A}$. The maximum displacement at a given temperature can be increased by increasing the length of the actuator. In present design, the optimized dimension of the Z-shaped actuator is $1.5 \mathrm{~mm}$ in length, $20 \mu \mathrm{m}$ in thickness, and $10 \mu \mathrm{m}$ in width.

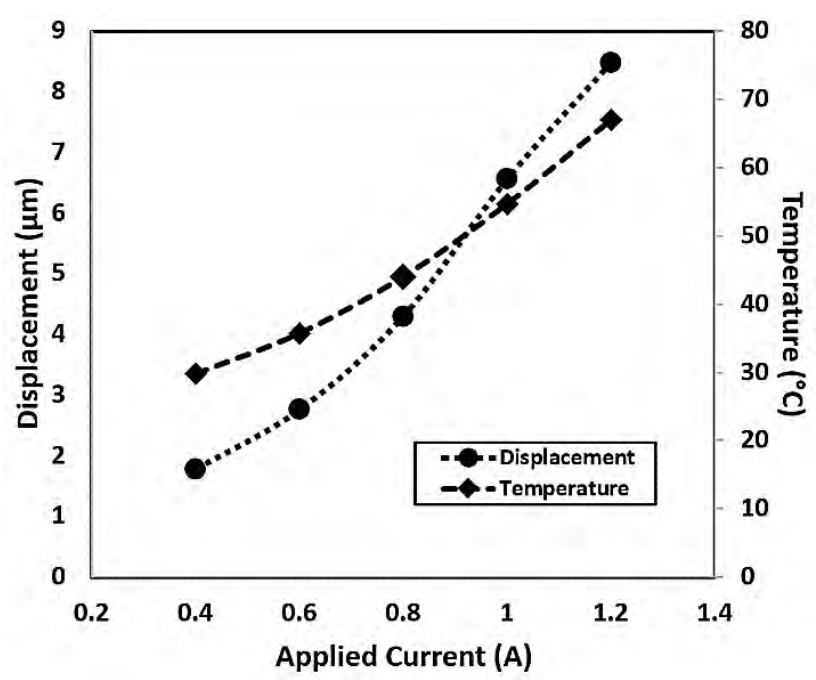

Fig. 15 Displacement and Temperature versus applied current

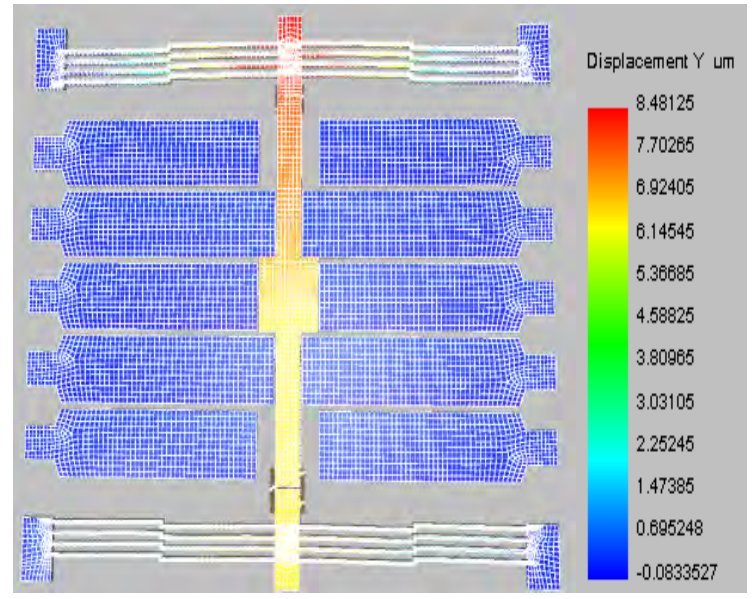

Fig. 16 Displacement with applied current of $1.2 \mathrm{~A}$ 


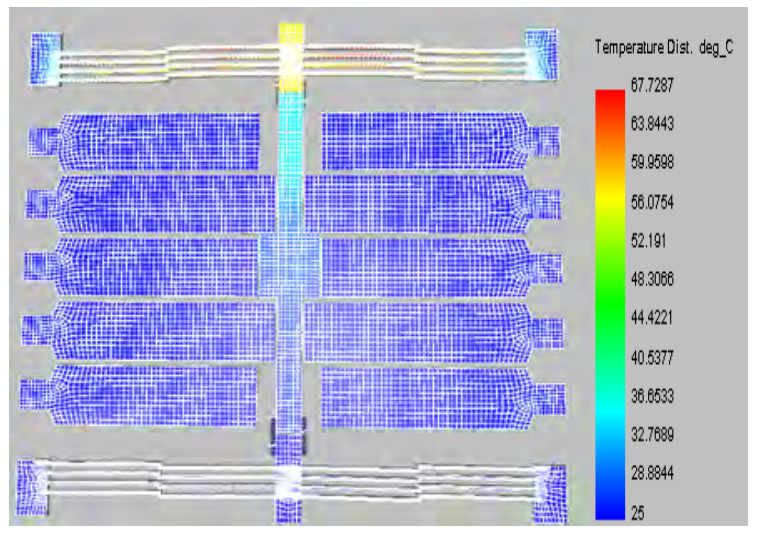

Fig. 17 Temperature distribution with applied current of $1.2 \mathrm{~A}$

Fig. 18 illustrates contact force with applied current of $1.2 \mathrm{~A}$. Over $1 \mathrm{mN}$ contact force is achieved in the design, which is sufficient to provide good contact quality between switch and the signal lines.

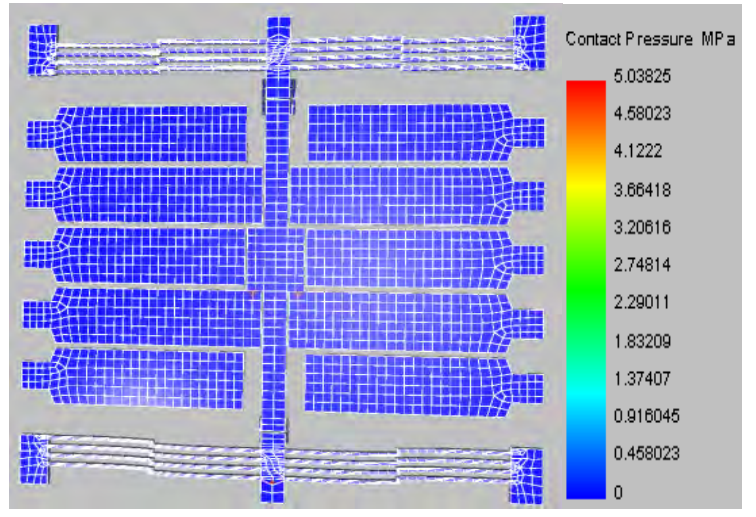

Fig. 18 Contact force with applied current of $1.2 \mathrm{~A}$

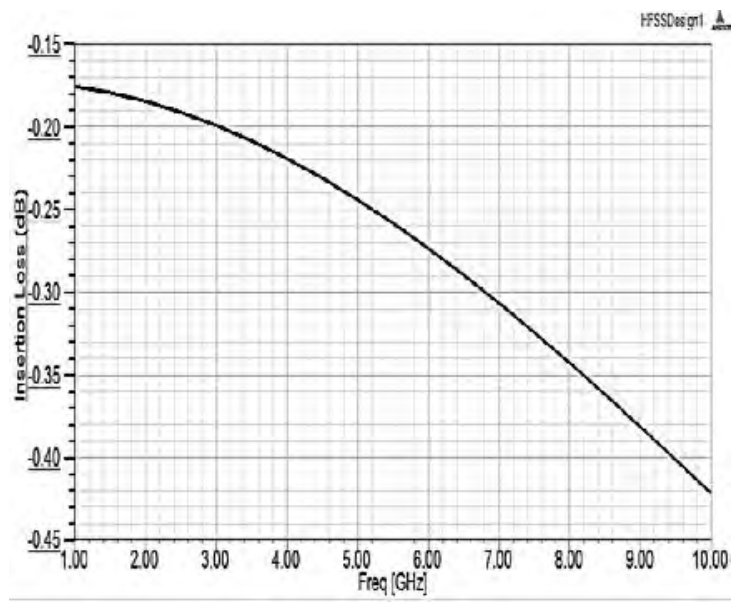

Fig. 19 ON state insertion loss for MEMS switch

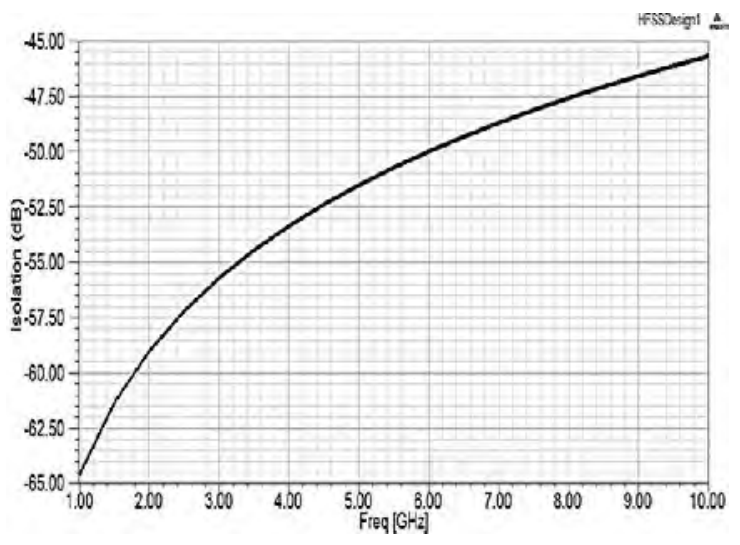

Fig. 20 OFF state isolation for MEMS switch

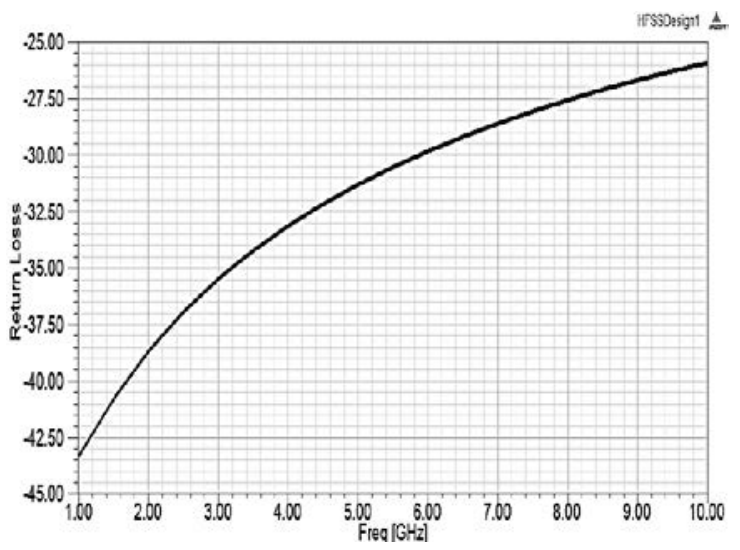

Fig. 21 ON state return loss for MEMS switch

Figures 19-21 illustrate S-parameters results for Z-shaped thermally actuated RF MEMS switch. The simulated RF results (insertion loss: $-0.42 \mathrm{~dB}$ at $10 \mathrm{GHz}$; isolation: $-64 \mathrm{~dB}$ at $1 \mathrm{GHz}$ and $-44 \mathrm{~dB}$ at $10 \mathrm{GHz}$; return loss: $43 \mathrm{~dB}$ at $1 \mathrm{GHz}$ and $-26 \mathrm{~dB}$ at $10 \mathrm{GHz}$ ) validate the strategy of suspending the structures on a low-resistivity substrate to obtain high performance RF switch in low-gigahertz frequency band.

\section{Conclusions}

The electrothermally actuated lateral contact microswitches for smart antenna applications are designed on a low resistivity silicon substrate using MetalMumps process. Electrothermal actuation is used as a driving principle as it provides higher contact force thus lower insertion loss. Measured insertion loss and isolation for Zshaped thermally actuated RF MEMS switch are $-0.42 \mathrm{~dB}$ and $-44 \mathrm{~dB}$ at $10 \mathrm{GHz}$. The return loss of the switch is 
also better than $-20 \mathrm{~dB}$ in the frequency range of $1 \mathrm{GHz}$ to $10 \mathrm{GHz}$. It is verified that RF performances can be improved by suspending the switch $25 \mu \mathrm{m}$ above the substrate. This technique would possibly allow the switch to be integrated with active circuitry manufactured on a low resistivity substrate in a system on chip concept, while sustaining good RF performances. The proposed MEMS switches will provide good performances as compared with PIN diode switches.

\section{References}

A. Cowen, B. Dudley,E. Hill, et al.,"MetalMUMPs Design Handbook", $\quad 1.0$. http://www.memsrus.com/ncmumps.metal.html

D. Girbau, N. Otegi, L. Pradell, and A. Lázaro (2006) "Study of intermodulation in RF MEMS variable capacitors," IEEE Trans. Microw. Theory Tech., vol. 54, no. 3, pp. $1120-1130$

D. Girbau, L. Pradell, A. Lazaro and A. Nebot (2007)“ Electrothermally Actuated RF MEMS Switches Suspended on a Low- Resistivity Substrate," Jornal of Microelectrothermal Systems, Vol. 16, No. 5, pp. $1061-1070$

H. A. C. Tilmans, W. de Raedt, and E. Beyne (2003) "MEMS for wireless communications: 'From RFMEMS components to RF-MEMS-SiP',' J. Micromech. Microeng., vol. 13, no. 4, pp. 139-163

J. Lu, David Thiel, Seppo Saario (2002) Multi-Objective Optimization in High Frequency Electromagnetics-An Effective Technique for Smart Mobile Terminal Antenna (SMTA) Design, IEEE Trans. on Magnetics, Vol. 38, No. 2, 701-704.

J. Dong and Ferreira P M 2008 Simultaneous actuation and displacement sensing for electrostatic drives $\mathrm{J}$. Micromech. Microeng. 18035011

J. K. Luo, Y. Q. Fu, J. A. Williams, Fellow, ASME, and W. I. Milne (2009) "Thermal Degradation of Electroplated Nickel Thermal Microactuators," Journal of Microelectrothermal Systems, Vol. 18, No. 6, pp. $1279-1287$
M.J. Sinclair (2000) "A High Force Low Area MEMS Thermal Actuator," IEEE Inter Society Conference on Thermal Phenomena, pp. 127-132

W. Shi, N.C. Tien, Member, IEEE, and Z. Li, Member (2007) IEEE “ a Highly Polysilicon as Isolation Material" Journal of Micromechanical Systems, Vol. 16, No. 5, PP. 1173-1184

W. H. Chen and et. Al (2007) "A Novel Planar Switched Parasitic Array Antenna With Steered Conical Pattern," IEEE Transactions on Antennas and Propagation, vol. 55, no. 6, pp. 183-187

Y. Zhu and Espinosa H D 2005 An electro-mechanical material testing system for in situ electron microscopy and applications Proc. Natl Acad. Sci. USA 102 14503-8.

Y. Zhu, S.O. Reza Moheimani and Mehmet 2012, "Bidirectional electrothermal actuator with Zshaped beams, J. IEEE sensors, Vol. 12, No. 7, 2508- 2509 